\title{
Scheduled Study Activity
}

National Cancer Institute

\section{Source}

National Cancer Institute. Scheduled Study Activity. NCI Thesaurus. Code C93356.

An activity that is anticipated to occur at some time in the future and has been assigned a time or date when that activity is to be performed. 